\title{
Adaptive Wavelet Collocation Method on the Shallow Water Model
}

Shanon M. Reckinger

Fairfield University, shanon.reckinger@fairfield.edu

Oleg V. Vasilyev

Baylor Fox-Kemper

Follow this and additional works at: https://digitalcommons.fairfield.edu/engineering-facultypubs

Copyright 2014 Elsevier

NOTICE: this is the author's version of a work that was accepted for publication in Journal of Computational Physics. Changes resulting from the publishing process, such as peer review, editing, corrections, structural formatting, and other quality control mechanisms may not be reflected in this document. Changes may have been made to this work since it was submitted for publication. A definitive version was subsequently published in Journal of Computational Physics, 271 (15), 342-359, August 2014. DOI: 10.1016/j.jcp.2014.03.043

\section{Peer Reviewed}

\section{Repository Citation}

Reckinger, Shanon M.; Vasilyev, Oleg V.; and Fox-Kemper, Baylor, "Adaptive Wavelet Collocation Method on the Shallow Water Model" (2014). Engineering Faculty Publications. 113.

https://digitalcommons.fairfield.edu/engineering-facultypubs/113

\section{Published Citation}

Reckinger, S. M., Vasilyev, O. V., Fox-Kemper, B., "Adaptive Wavelet Collocation Method on the Shallow Water Model", Journal of Computational Physics, 271 (15), 342-359, August 2014.

This item has been accepted for inclusion in DigitalCommons@Fairfield by an authorized administrator of DigitalCommons@Fairfield. It is brought to you by DigitalCommons@Fairfield with permission from the rightsholder(s) and is protected by copyright and/or related rights. You are free to use this item in any way that is permitted by the copyright and related rights legislation that applies to your use. For other uses, you need to obtain permission from the rights-holder(s) directly, unless additional rights are indicated by a Creative Commons license in the record and/or on the work itself. For more information, please contact digitalcommons@fairfield.edu. 


\title{
Adaptive Wavelet Collocation Method on the Shallow Water Model
}

\author{
Shanon M. Reckinger ${ }^{\mathrm{a}}$, Oleg V. Vasilyev ${ }^{\mathrm{b}}$, Baylor Fox-Kemperc \\ ${ }^{a}$ Fairfield University \\ Department of Mechanical Engineering \\ 1073 North Benson Rd \\ Fairfield, CT 06824 \\ Tel.: +001-203-254-4000-2527 \\ shanon.reckinger@fairfield.edu \\ ${ }^{b}$ University of Colorado, Boulder \\ Department of Mechanical Engineering, UCB 427 \\ Boulder, CO 80309 \\ Tel.: +001-303-492-4717 \\ oleg.vasilyev@colorado.edu \\ ${ }^{c}$ Brown University \\ Department of Geological Sciences \\ Providence, RI 02912 \\ Tel.: +001-401-863-1000 \\ Baylor@brown.edu
}

\begin{abstract}
This paper presents an integrated approach for modeling several ocean test problems on adaptive grids using novel boundary techniques. The adaptive wavelet collocation method solves the governing equations on temporally and spatially varying meshes, which allows higher effective resolution to be obtained with less computational cost. It is a general method for the solving a large class of partial differential equations, but is applied to the shallow water equations here. In addition to developing wavelet-based computational models, this work also uses an extension of the Brinkman penalization method to represent irregular and non-uniform continental boundaries. This technique is used to enforce no slip boundary conditions through the addition of a term to the field equations. When coupled with the adaptive wavelet collocation method, the flow near the boundary can be well resolved. It is especially useful for simulations of boundary currents and tsunamis, where flow and the boundary is important, thus, those are the test cases presented here.
\end{abstract}


Keywords: Immersed Boundary Methods, Shallow Water Equations, Adaptive Mesh Refinement, Wavelet Collocation, Complex Geometry

\section{Introduction}

Two numerical techniques are used for the solution of the shallow water equations for a variety of test cases. The first technique is the adaptive wavelet collocation method, which is a general solution technique for solving differential equations on adaptive, collocated grids. This method uses wavelet properties to determine, on the fly, significant points needed for an accurate solution. The second is the shallow water formulation of the Brinkman penalization method, which is an immersed boundary method used to define solid boundaries of arbitrary complexity. Four test cases are presented to validate these methods. Test Cases I and II study the effect of using the adaptive wavelet collocation method on the shallow water model. These cases include rotating waves on a beta-plane and a wind-driven single gyre in a square basin. Test Cases III and IV study the effect of using the combination of the wavelet method and Brinkman penalization. These cases include a North Atlantic simulation and a tsunami simulation.

\section{Adaptive Wavelet Collocation Method}

The adaptive wavelet collocation method is a general method for the solution of a large class of linear and nonlinear partial differential equations (Vasilyev and Bowman, 2000; Vasilyev, 2003; Vasilyev and Kevlahan, 2005; Regele and Vasilyev, 2009). The method has already been successfully applied in wide range of fluid mechanics problems, e.g., Vasilyev et al. 1997; Vasilyev and Kevlahan 2002; Kevlahan et al. 2007; Reckinger et al. 2010; Schneider and Vasilyev 2010. In this section, the methodology is briefly reviewed.

The benefit of using wavelets is that they are localized in both space and time. They are ideal for use in complex flows where localized structures exist in the solution. The wavelet collocation method takes advantage of wavelet compression properties (Vasilyev et al., 1997). Functions with localized structures or regions with sharp transitions are well compressed using wavelet decomposition. This compression is achieved by keeping only the wavelets with coefficients that are greater than an a priori threshold parameter. This allows high resolution computations to be carried out only in the 
regions where it is necessary. It also allows a solution to be obtained on a near optimal grid for a given accuracy. Figure 15 shows an example of a simulation of the 2010 Chile Tsunami using the Adaptive Wavelet Collocation Method to solve the shallow water equations with real variable bathymetry (ETOPO2, 2010). The right side of the figure shows that the grid is localized near the tsunami and near all the continental boundaries.

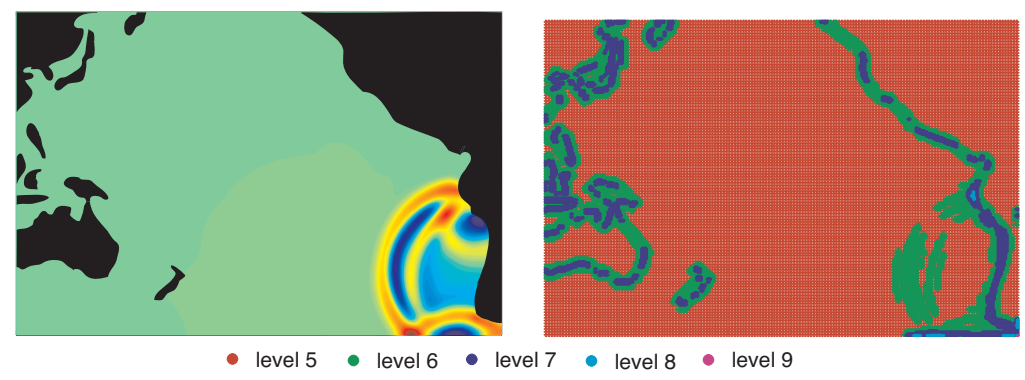

Figure 1: Results from 2010 Chile Tsunami simulation, with sea surface height on the left and the adaptive grid (colored by level) on the right using the Adaptive Wavelet Collocation Method.

Any function $u(\mathbf{x})$ in an $n$-dimensional space can be decomposed as (Chui, 1997; Daubechies, 1992; Mallat, 1998)

$$
u(\mathbf{x})=\sum_{\mathbf{k} \in \mathcal{K}^{0}} c_{\mathbf{k}}^{0} \phi_{\mathbf{k}}^{0}(\mathbf{x})+\sum_{j=0}^{+\infty} \sum_{\mu=1}^{2^{n}-1} \sum_{\mathbf{l} \in \mathcal{L}^{\mu, j}} d_{\mathbf{l}}^{\mu, j} \psi_{\mathbf{l}}^{\mu, j}(\mathbf{x}),
$$

where $\phi_{\mathbf{k}}^{0}(\mathbf{x})$ are scaling functions on the lowest level of resolution and $\psi_{\mathbf{l}}^{\mu, j}(\mathbf{x})$ are the wavelet basis functions. Also, $c_{\mathbf{k}}^{0}$ and $d_{\mathbf{l}}^{\mu, j}$ are the scaling and wavelet coefficients, respectively. The wavelet coefficients, $d_{1}^{\mu, j}$, are small except near areas with large gradients. Equation 1 can be decomposed into two terms whose wavelet coefficients are above and below a chosen threshold parameter $\epsilon$,

$$
u(\mathbf{x})=u_{\geq}(\mathbf{x})+u_{<}(\mathbf{x}),
$$

where

$$
u_{\geq}(\mathbf{x})=\sum_{\mathbf{k} \in \mathcal{K}^{0}} c_{\mathbf{k}}^{0} \phi_{\mathbf{k}}^{0}(\mathbf{x})+\sum_{j=0}^{+\infty} \sum_{\mu=1}^{2^{n}-1} \sum_{\substack{1 \in \mathcal{L}^{\mu, j} \\\left|d_{\mathbf{l}}^{\mu, j}\right| \geq \epsilon\|u\|}} d_{\mathbf{l}}^{\mu, j} \psi_{\mathbf{l}}^{\mu, j}(\mathbf{x})
$$




$$
u_{<}(\mathbf{x})=\sum_{j=0}^{+\infty} \sum_{\mu=1}^{2^{n}-1} \sum_{\substack{\mathbf{1} \in \mathcal{L}^{\mu, j} \\\left|d_{1}^{\mu, j}\right|<\epsilon|| u \|}} d_{\mathbf{l}}^{\mu, j} \psi_{\mathbf{l}}^{\mu, j}(\mathbf{x})
$$

Donoho (1992) was able to show that for a regular function the error is bounded as

$$
\left\|u(\mathbf{x})-u_{\geq}(\mathbf{x})\right\| \leq C_{1} \epsilon\|u\|
$$

which means that the number of grid points needed to solve a numerical problem can be significantly reduced while still retaining a prescribed level of accuracy determined by the threshold parameter $\epsilon$.

In the wavelet collocation method there is a one-to-one correspondence between grid points and wavelets. This makes calculation of nonlinear terms simple, and allows the grid to adapt automatically to the solution at each time step by adding or removing wavelets. In addition to the points with significant wavelet coefficients, several other checks are performed to ensure the resolution is sufficient for the given simulation. The way the method works is, at the beginning of each time step, the wavelet coefficients are calculated. Wavelets with significant coefficients are identified, which are those larger than $\epsilon$. Next, to account for the evolution of the solution over time, the nearest neighbor wavelet coefficients in position and scale are also added (Liandrat and Tchamitchian, 1990). After these significant and adjacent points are kept, the wavelets that are below the threshold $\epsilon$ and are not in the adjacent zone are removed. It can be shown that the $L_{\infty}$ error for this approximation is bounded by $\epsilon$. This allows the grid to automatically follow the evolution of the solution. Then, reconstruction points are added, which are points needed to compute the wavelet transforms. Lastly, ghost points are added, these are points needed to calculate spatial derivatives. The spatial derivatives are calculated using finite differences. Since our simulations uses second generation wavelets (Sweldens, 1998), the order of the wavelet (and also finite difference) can be easily varied.

Figure 2 shows a one-dimensional example of a solution (top) and its adaptive grid (bottom). The vertical lines show the magnitude of the wavelet coefficients at each location in space for each level of resolution. It is clear that at the location in the center of the $x$-axis where the solution has a sharp gradient, there is localized refinement on the grid.

There are some additional computational costs associated with the use of the adaptive multi-resolution wavelet method. Currently, the cost per grid 

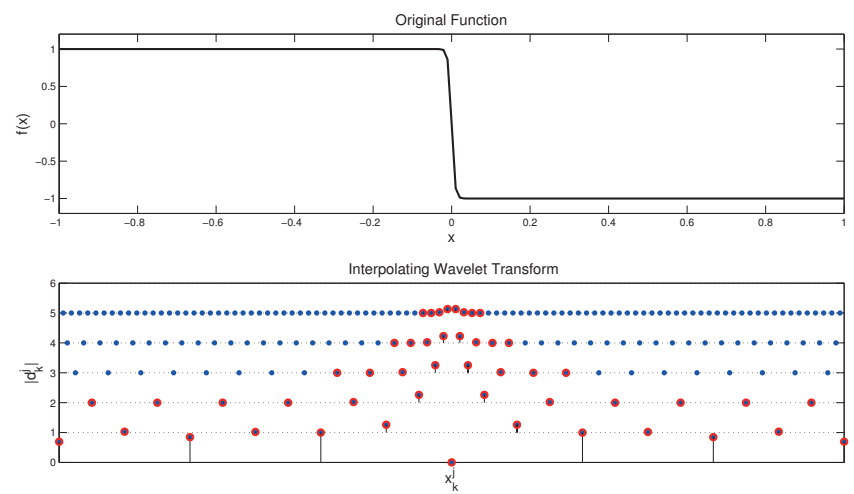

Figure 2: A one dimensional example of grid adaptation using the Adaptive Wavelet Collocation Method. Top plot shows function. Bottom plot shows wavelet coefficients, with height of each dot indicating magnitude of the coefficient.

point is approximately three to five times greater than the cost of a standard non-adaptive method. However, in cases of large compressions (Kevlahan and Vasilyev, 2005), the compression greatly outweighs this cost. There is also some memory savings associated with using adaptive methods, which allows higher resolution simulations with the same computational resources.

In summary, the dynamically adaptive wavelet collocation method is an adaptive, variable order method for solving partial differential equations with localized structures that change their location and scale in space and time. Since the computational grid automatically adapts to the solution (in position and scale), we do not have to know a priori where the regions of high gradients or structures exist. The wavelet method was recently extended for massively parallel architectures (Nejadmalayeri, 2012). The simulations reported in this paper use the parallel adaptive wavelet collocation solver with dynamic load balancing.

\section{Shallow Water Formulation of the Brinkman Penalization Method}

The numerical technique used for representation of continental and irregularly shaped boundaries is an extension of the Brinkman penalization technique developed for use in the shallow water equations (Reckinger et al., 2012). This method was developed through the extension of the compressible formulation of Brinkman penalization developed by Liu and Vasilyev (2007). 
Adapting this Brinkman penalization to the shallow water equations gives

$$
\begin{gathered}
\frac{\partial \eta}{\partial t}=-\left[1+\left(\frac{1}{\phi}-1\right) \chi\right] \nabla \cdot(\eta \mathbf{u}), \\
\frac{\partial \mathbf{u}}{\partial t}+\mathbf{u} \cdot \nabla \mathbf{u}+\frac{1}{R o} f^{\prime} \hat{\mathbf{k}} \times \mathbf{u}=-\frac{1}{F r^{2}} \nabla \eta-\frac{\chi}{\eta_{\text {pen }}} \mathbf{u},
\end{gathered}
$$

where $R_{0}=U / L f, f^{\prime}=1+\beta y$, and $F r=U / \sqrt{g H}$ following the nondimensionalization following Pedlosky (1987). The two added parameters for penalization are Brinkman penalization parameter, $\eta_{\text {pen }} \ll 1$, and the porosity parameter, $\phi \ll 1$.

$$
\chi(\mathbf{x}, t)= \begin{cases}1 & \text { if } \mathbf{x} \in O_{i}(\mathbf{x}), \\ 0 & \text { otherwise }\end{cases}
$$

which is called a masking function. $O_{i}(\mathbf{x})$ is the set of all obstacles, or, in the case of shallow water ocean simulations, is the continental boundary. The terms added for penalization are colored red in Equations 6 and 7. The term added to the momentum equation enforces the no slip boundary condition. Since $\eta_{\text {pen }} \ll 1$, the penalized term dominates the equation and the solution is a decaying exponential, where $\eta_{\text {pen }}$ is a time scale which controls the rate of decay to zero within the boundaries. The term added to the continuity equation ensures conservation of mass. It does so by modeling the Brinkman zone as a porous medium. As the $\lim \phi \rightarrow 1$, the porous medium approaches the limit of a solid boundary.

It is important to note that the shallow water equations are mathematically equivalent to the compressible gas dynamic equations in the adiabatic or isothermal limit. Since the compressible formulation of the Brinkman penalization was developed for the full compressible gas dynamic equations, a unique treatment of the added parameters is necessary for the shallow water

formulation of the Brinkman penalization method. A detailed analysis of these equations and the numerical testing of the parameters can be found in Reckinger et al. (2012). All of the guidelines set out for setting parameters has been followed in the test cases presented here.

\section{Testing and Validation}

As a first step of investigating ocean circulation modeling using the adaptive wavelet method, the shallow water equations are chosen. There are 
several reasons. First, it is necessary to test the wavelet methodology in two dimensions. Therefore, the two dimensional modeling could take two directions: the barotropic vorticity equation or the shallow water equations. The shallow water governing equations were chosen because they represent a wider variety of wave modes for testing. Also, by using the shallow water equations, the shallow water formulation of Brinkman penalization could be used.

Four test cases will be presented in this section. The first test case is rotating waves on a beta-plane, which demonstrates the advantage of having an on-the-fly adaptive mesh. The second test case is a single wind-driven gyre in a square basin, which shows a range of grid resolutions. Results from this test case are compared to published results to validate the adaptive wavelet collocation method accuracy. Both of these first two cases focus on verifying the wavelet method and do not use the shallow water formulation of Brinkman penalization, since they both have straight vertical, traditional boundaries implemented directly as edges of the computational domain. The third case is a simplified ocean gyre in the North Atlantic, with realistic continental boundaries. The fourth case is a simulation of the 2010 Chile tsunami with realistic continental boundaries and bathymetry. These last two cases give some insight on the strength of coupling the adaptive wavelet collocation method and the Brinkman penalization method used in ocean circulation problems.

The shallow water equations are non-dimensionalzed in two different ways for the four test cases. Test Cases I and IV use the following nondimensionalization (the other non-dimensionalization will be presented in a later section),

$$
\begin{gathered}
\frac{\partial \eta}{\partial t}=-\nabla \cdot(\eta \mathbf{u}) \\
\frac{\partial \mathbf{u}}{\partial t}+\mathbf{u} \cdot \nabla \mathbf{u}+\frac{1}{R o} f^{\prime} \hat{\mathbf{k}} \times \mathbf{u}=-\frac{1}{F r^{2}} \nabla \eta+\frac{1}{R e} \nabla^{2} \mathbf{u}+\mathbf{F}_{\text {wind }}
\end{gathered}
$$

In these non-dimensional equations $\eta$ is the sea surface height, $u$ and $v$ are the horizontal components of velocity, $f^{\prime}=1+\beta y$ is the Coriolis force, $R o \sim U / f_{0} L$ is the Rossby number, $U$ is the velocity scale, $L$ is the length scale, $F r=\sqrt{g H} / U$ is the Froude number, $H$ is the depth scale, $\mathbf{F}_{\text {wind }}$ is the analytic wind forcing function, $T \sim L / U$ is the time scale, and $R e=U L / \nu_{e}$ is the Reynolds number. The $R e$ is not representative of molecular viscosity. It is used as a parameterization of turbulent processes (an eddy viscosity 
turbulent model). It models not only the effects of eddies, but also all the processes which would remove vorticity from the basin at the boundary, such as bottom topography effects.

\subsection{Test Case I: Rotating Waves on a Beta-Plane}

Test Case I focuses on demonstrating the advantage of using the adaptive wavelet collocation method to solve problems with dynamics that change rapidly in space and time. For this, rotating waves on a beta-plane are studied. Equations 9 and 10 are used for this test case. Rotating waves can be best described by linear wave dynamics of an inviscid, homogenous fluid under rotation on a beta plane. The dimensional equations in the $R o \rightarrow 0$ and $R e \rightarrow \infty$ are

$$
\begin{gathered}
\frac{\partial \eta}{\partial t}=-H\left(\frac{\partial u}{\partial x}+\frac{\partial v}{\partial y}\right), \\
\frac{\partial u}{\partial t}-\left(f_{0}+\beta_{0} y\right) v=-g \frac{\partial \eta}{\partial x} \\
\frac{\partial v}{\partial t}+\left(f_{0}+\beta_{0} y\right) u=-g \frac{\partial \eta}{\partial y} .
\end{gathered}
$$

The first dispersion relation that can be derived from this set of equation is

$$
\omega=-\beta_{0} R^{2} \frac{l}{1+R^{2}\left(l^{2}+m^{2}\right)},
$$

where $\omega$ is the frequency, $l$ and $m$ are wavenumbers, and $R=\sqrt{g H} / f_{0}$ is the deformation radius. These waves are called Rossby or planetary waves. They are very slow waves, which always have negative zonal phase speed, $c_{\mathrm{x}}=-\beta_{0} R^{2} /\left[1+R^{2}\left(l^{2}+m^{2}\right)\right]$. These waves have a phase propagation to the west. These waves only arise when there are spatial variations in background vorticity (they are not present for f-plane or no rotation).

There are several other modes that can be described by Equations 11 13. Ignoring the beta effect, the following dispersion relation results,

$$
\omega=\sqrt{f^{2}+g H k^{2}} .
$$

This is the dispersion relation for inertia-gravity waves, or Poincare waves. The name comes from exhibiting a mixed behavior of both gravity waves and inertial oscillations. For large wavenumbers $\left(k^{2} \gg f^{2} / g H\right)$, or wavelengths much shorter than the deformation radius, the waves become classical gravity 
waves $(\omega=k \sqrt{g H})$. This is because the waves are too short to be affected by the rotation of the earth. Also, in the opposite limit of low wavenumbers $\left(k^{2} \ll f^{2} / g H\right)$, the rotation effects dominate $(\omega=f)$, and the flow is virtually all uniform with each fluid particle moving in unison, each describing a circular inertial oscillation.

[PRINT VERSION: Figure 3 shows a series of snapshots in time of the rotating wave.] [ONLINE VERSION: Video 4.1 shows an animation of the rotating waves through time.] The sea surface height is initialized as a Gaussian bump. At the beginning of the simulation, gravity waves are created, propagate out, hit the no slip boundary walls, and reflect back and forth until they eventually dissipate as a result of numerical dissipation. At this same time, the entire flow is exhibiting behavior of inertial oscillations with the velocity vectors spiraling cyclonically. This behavior is most evident in the beginning of simulation. These initial waves are all fairly fast and are created and die on the gravity wave timescale of the simulation. After a long time, only the Rossby wave mode remains, which is manifest as a nearly geostrophically balanced high surface that propagates slowly westward. The clockwise circulation pattern sits in the center of the domain and slowly creeps west until it eventually hits the western wall.

\section{PLACE Vid1.mov HERE.}

The time series of rotating waves demonstrates the advantage of the adaptive wavelet method. The grid is constantly adapting to whatever modes are present in the simulation. Initially, when the gravity waves are created, the grid has many points to properly resolve those gravity waves. They eventually dissipate out, leaving the grid to adapt only on the slowly westward moving clockwise circulation pattern (and to the boundaries until they are no longer needed).

\subsubsection{Wavelet Compression}

The effective resolution for the simulation is $1024 \times 1024$. [PRINT VERSION: The percentage of points used for each snap shot (from left to right, top the bottom) is $3.37 \%, 5.39 \%, 3.78 \%, 1.35 \%, 0.46 \%, 0.55 \%$.] [ONLINE VERSION: The percentage of points used at any give time in the simulation ranges from $0.55 \%$ to $5.39 \%$.] That is a maximum compression rate of $99.56 \%$ and a minimum of $94.61 \%$. 

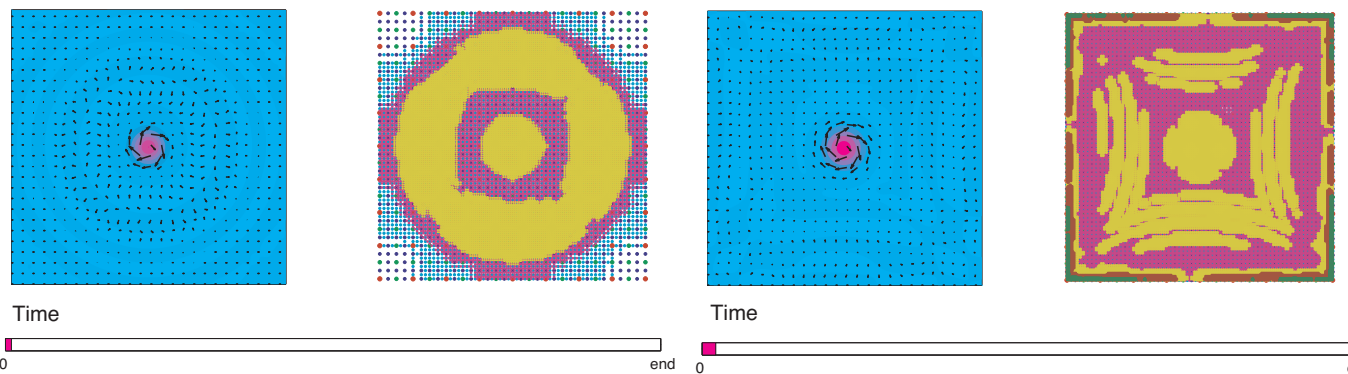

Time

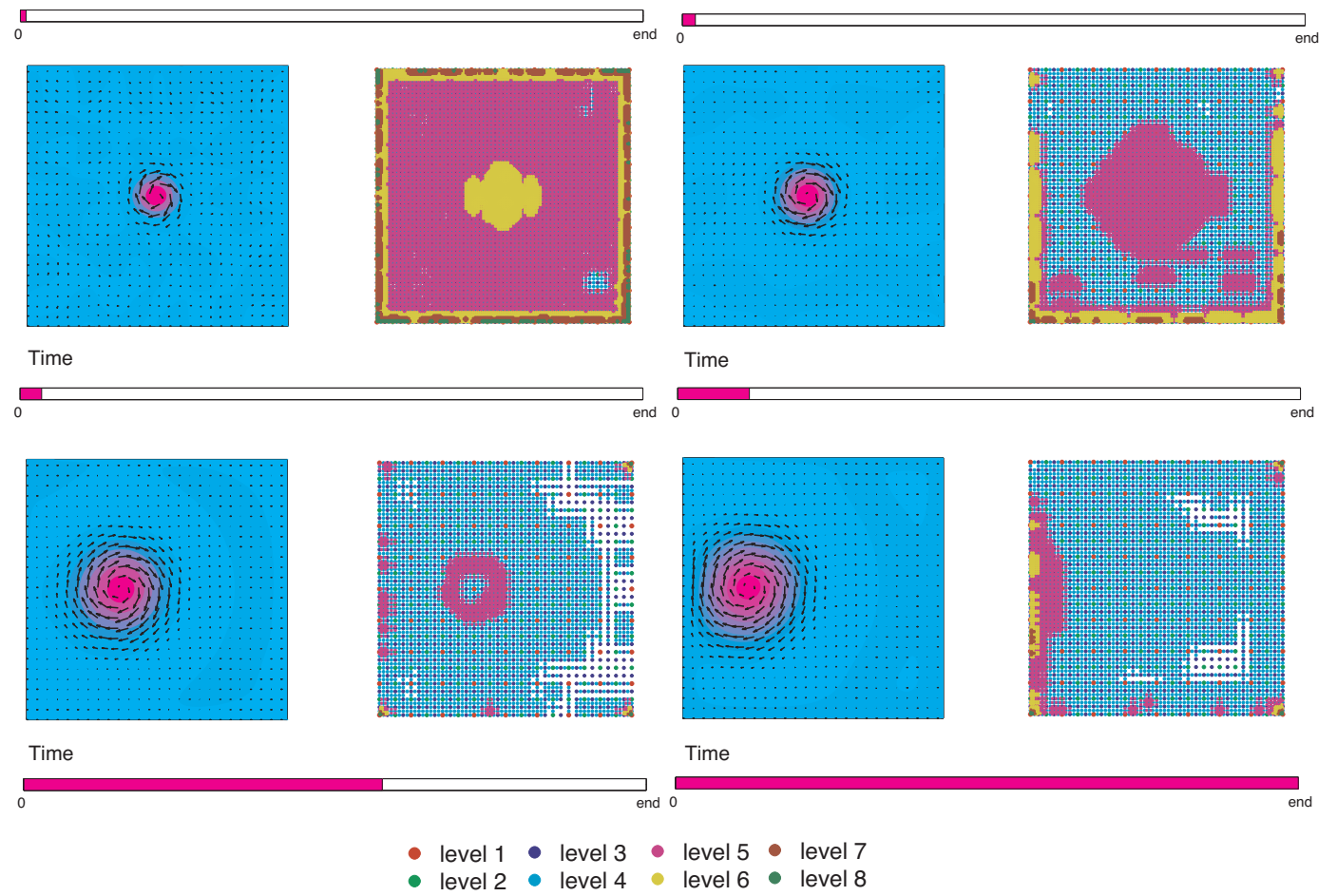

Figure 3: Results and their associated adaptive grids for the rotating waves under the beta plane approximation test problem.

\subsection{Test Case II: Single Wind-Driven Gyre}

In order to validate the accuracy of the wavelet method in a more nonlinear scenario, a comparison study is done based on the work by Fox-Kemper and Pedlosky (2004). The model test case is a rigid lid, homogeneous density, single-gyre ocean model on a beta plane with viscosity. The single gyre wind forcing is intended to roughly model a northern hemisphere subtropical gyre. Double gyre models are also often used. The importance of the double-gyre model is discussed in Fox-Kemper (2004). 
To directly compare test cases, the governing equations are made dimensionless in such a way that the boundary layer width parameters are explicit. The Fox-Kemper model solves the barotropic vorticity equations. The barotropic vorticity equations are a subset of the shallow water equations in the infinite Froude number limit which filters out the gravity waves. The derivatives taken to derive the barotropic vorticity equation remove the effect of hydrostatic pressure, thus, eliminating shallow water gravity wave modes from the solution. For comparison, the barotropic vorticity equation model used in Fox-Kemper and Pedlosky (2004) is

$$
\begin{gathered}
\frac{\partial \zeta}{\partial t}+\delta_{I}^{2}\left(\frac{\partial \Psi}{\partial x} \frac{\partial \zeta}{\partial y}-\frac{\partial \Psi}{\partial y} \frac{\partial \zeta}{\partial x}\right)+\frac{\partial \Psi}{\partial x}=-\sin (\pi y)+\nabla \delta_{M}^{3} \nabla \zeta \\
\zeta=\nabla^{2} \Psi .
\end{gathered}
$$

To derive the shallow water equations with the equivalent non-dimensionalization, start with the dimensional shallow water equations, including all the forcing terms,

$$
\begin{gathered}
\frac{\partial \eta^{\star}}{\partial t^{\star}}+\nabla \cdot\left(\eta^{\star} \mathbf{u}^{\star}\right)=0, \\
\frac{\partial \mathbf{u}^{\star}}{\partial t^{\star}}+\mathbf{u}^{\star} \cdot \nabla \mathbf{u}^{\star}+f \hat{\mathbf{k}} \times \mathbf{u}^{\star}=-g \nabla \eta^{\star}+\nu \nabla^{2} \mathbf{u}^{\star}-\frac{f_{0} L W_{E}}{D \pi} \cos \left(\frac{\pi y^{\star}}{L}\right) .
\end{gathered}
$$

The non-dimensionalized quantities are

$$
\begin{array}{cc}
t^{\star} & =\frac{t}{\beta L}, \quad x^{\star}=L x, \quad y^{\star}=L y, \\
\eta^{\star} & =D \eta, \quad f=f_{0}+\beta L y^{\star}, \quad u^{\star}=\frac{W_{E} f_{0}}{\beta D} u,
\end{array}
$$

where the time scale is approximately several hours, and the velocity is scaled in such a way that the streamfunction of the Sverdrup solution would have a maximum of $\Psi=1$ were it to fill the dimensions of the basin (as is determined by Fox-Kemper and Pedlosky (2004)). Plugging in all the quantities gives the full set of non-dimensional equations,

$$
\begin{gathered}
\frac{\partial \eta}{\partial t}+\delta_{I}^{2} \nabla \cdot(\eta \mathbf{u})=0 \\
\frac{\partial \mathbf{u}}{\partial t}+\delta_{I}^{2} \mathbf{u} \cdot \nabla \mathbf{u}+\left(\frac{1}{P l}+y\right) \hat{\mathbf{k}} \times \mathbf{u}=-\frac{\delta_{I}^{2}}{F r^{2}} \nabla \eta+\delta_{M}^{3} \nabla^{2} \mathbf{u}-\frac{1}{\pi} \cos (\pi y) \hat{\mathbf{i}},
\end{gathered}
$$

where,

$$
\begin{array}{ll}
\delta_{I}^{2}=\frac{W_{E} f_{0}}{\beta^{2} D L^{2}}, & \delta_{M}^{3}=\frac{\nu}{\beta L^{3}}, \\
F r=\frac{U}{\sqrt{g D}}, & P l=\frac{\beta L}{f_{0}},
\end{array}
$$


where $U=\left(W_{E} f_{0}\right) /(\beta D)$. In this formulation, the parameters, $\delta_{I}$ and $\delta_{M}$, are the Charney (1955) and Munk (1950) boundary layer scales, respectively. The viscosity parameter, $\delta_{M}^{3}$, is proportional to the dimensional viscosity and plays a similar role in the nondimensional equations. The Reynolds number of the boundary layer is defined as $\delta_{I}^{3} / \delta_{M}^{3}$, although others use a Reynolds number approximate to a basin-wide flow, defined as $\delta_{I}^{2} / \delta_{M}^{3}$. For all results presented, the value of $\delta_{\mathrm{I}}$ is fixed at 0.02 . This value corresponds to $80 \mathrm{~km}$ inertial boundary current scale in a $4000 \mathrm{~km}$ basin with a velocity scale of $0.1 \mathrm{~m} / \mathrm{s}$.

In the barotropic vorticity equation, there are only two non-dimensional constants to be set, $\delta_{I}$ and $\delta_{M}$. In the shallow water equations, two additional constants need to be set, including the Froude number, Fr, and the Planetary number, $P l$. These parameters are chosen such that the limit of the barotropic vorticity model is approached without a requirement for unduly short time steps to resolve gravity waves $(F r=0.001$ and $P l=0.1)$.

The zonal boundaries are at $x=0$ and $x=1$ and the meridional boundaries are at $y=0$ and $y=1$. The eastern and western boundaries have no slip boundary conditions $(u=0$ and $v=0)$. The northern and southern boundaries have slip boundary conditions $(v=0$ and $\partial u / \partial x=0)$.

\subsubsection{Analytic Initial Conditions}

For wind-driven, single gyre problems in a square basin, zero initial conditions are often used. Since zero initial conditions satisfy the boundary conditions and are solutions to the governing equations without forcing, they are often the best choice. However, when working with adaptive grids, resolving the initial boundary layer that is created by the instantaneous forcing can be expensive. To avoid the initial resolution requirement, analytic functions are occasionally used for initial conditions,

$$
\begin{gathered}
\eta=\sin (\pi \mathrm{y})[\cos (\pi(2 \mathrm{x}+1))], \\
u=-\cos (\pi \mathrm{y})[\cos (\pi(2 \mathrm{x}+1))], \\
v=-2 \pi \sin (\pi \mathrm{y})[\sin (\pi(2 \mathrm{x}+1))] .
\end{gathered}
$$

All variables are approximately $\mathrm{O}(1)$ but can be rescaled as needed. This solution satisfies the boundary conditions discussed above. They also reduce the computational cost of developing and resolving a boundary layer. These 
types of initial conditions are not required, but do speed up the time it takes to reach a steady circulation pattern. Figure 4 shows $\eta, u, v$ in graphical forms.
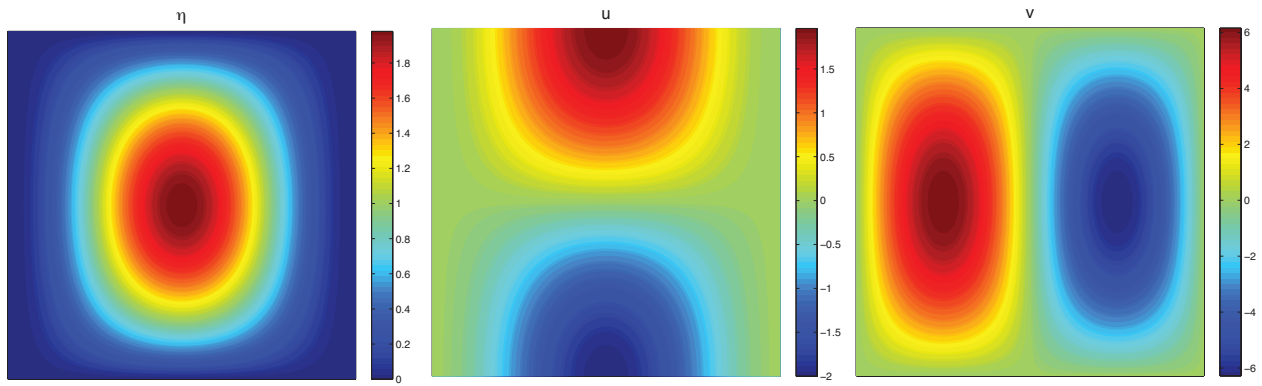

Figure 4: Analytic initial conditions for wind-driven single gyre problems.

\subsubsection{Results}

In order to test the accuracy and stability of the wavelet method with the boundary layer non-dimensionalization model (Equations 21 and 22), two different cases are considered. Using the boundary layer Reynolds number discussed earlier, $R e=0.5$ and 1 cases are compared to the results published in Fox-Kemper and Pedlosky (2004) (see Figure 5).

Since all the Fox-Kemper results are given in the form of streamfunctions, all of the results from the wavelet model are presented in terms of sea surface height, which for the shallow water equations in geostrophic balance (thus, any wind-driven gyre) is nearly equivalent to streamfunction. When flow is in geostrophic balance, the dominating balance is

$$
\mathbf{f} \times \mathbf{u}=-g \nabla \eta .
$$

When streamfunction definitions are substituted in $(u=\partial \psi / \partial y$ and $v=$ $-\partial \psi / \partial x)$, the following relationship is obtained, $\psi=-(g / f) \eta$. Thus, qualitatively, the streamfunctions and sea surface height can be used interchangeably for flow in geostrophic balance.

The series of plots from Fox-Kemper and Pedlosky (2004) in Figure 5 are the time-averaged results from the last half of each of the runs. The time unit, $(\beta L)^{-1}$, is approximately equivalent to about 3.5 hours. They are run to an integration time of about 10,000 time units, which is approximately 4 years. Additionally, the results from Fox-Kemper and Pedlosky (2004) uses a resolution of $257 \times 257$ Chebyshev modes. 
$\operatorname{Re}=0.2$
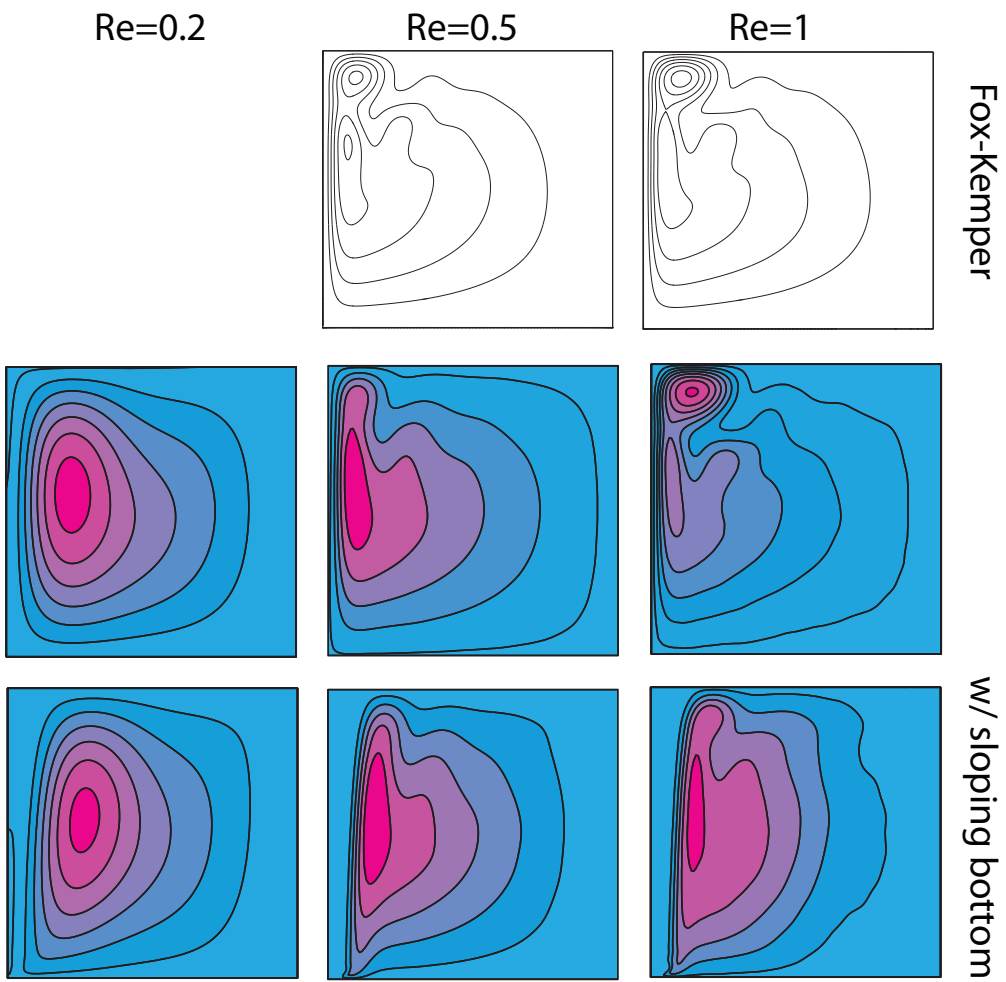

Figure 5: Qualitative Comparison of results from Fox-Kemper and Pedlosky (2004) to cases run using the wavelet method. All solutions are time-averaged over the last half of the simulation time.

The results from all cases $(R e=0.2,0.5,1)$ using the adaptive wavelet method are in excellent agreement with the results found in Fox-Kemper and Pedlosky (2004). A quantitative comparison was also done on two cases $(R e=0.5,1)$. Since the streamfunction and the sea surface height are not exactly equivalent, the zonal and meridional velocities will be compared instead. This quantitative comparison was done for two different Reynolds number and for both horizontal velocity components for each Reynolds number, which results in four error calculations and are shown in Figure 6.

The quantitative comparison in Figure 6 shows that the Fox-Kemper and the adaptive wavelet code results are in excellent agreement. The plots of relative error shows that the large difference between the two cases are in the center of the strong cyclones and anti-cyclones. Since the Fox-Kemper results were obtained using the barotropic vorticity equation and the results from 
the adaptive wavelet code were obtained using the shallow water equations, these differences are exactly what is expected. Cyclones should be a bit stronger when using the shallow water model and that anticyclones should be a bit weaker compared to barotropic vorticity equations. Table 1 shows the relative $\mathrm{L}_{2}$ errors for each of these cases. Considering the differences between the two sets of equations solved, the interpolation error associated with getting each set of data on the same grid, and the error associated with getting both data in terms of velocities, these $\mathrm{L}_{2}$ errors are expected.

\begin{tabular}{|l|c|c|}
\hline & $R e=0.5$ & $R e=1$ \\
\hline Relative $\mathrm{L}_{2}$ Error for $\mathbf{u}$ & 0.0143 & 0.0250 \\
Relative $\mathrm{L}_{2}$ Error for $\mathbf{v}$ & 0.0072 & 0.0286 \\
\hline
\end{tabular}

Table 1: The relative $\mathrm{L}_{2}$ errors between the adaptive wavelet collocation method and FoxKemper results. $\mathrm{L}_{2}$ errors were calculated for two different Reynolds numbers for both horizontal velocities.

Additionally, there are two more non-dimensional constants present in the shallow water formulation of the non-dimensionalization of the two sets of equations. These parameters include $F r$ and $P l$ (as seen from Equations 21 and 22). One way to check the accuracy of the adaptive wavelet collocation method results is to make sure they are approximately $\mathrm{O}(\mathrm{Fr})$ or $\mathrm{O}(\mathrm{Pl})$, which ever is larger. In the two cases used for direct comparison the $\mathrm{Fr}=0.001$ and $P l=0.1$.

\subsubsection{Time Step Restriction}

The Fox-Kemper model solved the barotropic vorticity equations. Since gravity waves are filtered out in these equations, a larger time step is allowed, since the timescale of the gravity waves does not need to be resolved. For the shallow water equations, there is a very restrictive time step to satisfy the CFL condition. Several different techniques are investigated to mitigate this issue.

One technique is to resolve the gravity waves generated initially as the gyre is first formed from initial conditions. Then, once all the generated gravity waves are dissipated, increase the time step and no longer resolve the gravity wave timescale. This technique is often used successfully with the compressible Navier-Stokes equations, to deal with the acoustic waves that are generated. However, with the shallow water equations, stability issues arise if the CFL condition is not satisfied at any point in the simulation. 
Even after the solution reaches a somewhat steady state (and even if the solution is well behaved and laminar), the gravity wave CFL condition needs to be satisfied. It is determined that the fast modes (gravity wave modes, inertia-gravity wave modes, etc.) are too easily and often excited to get away with not satisfying the CFL requirement.

The second technique is to not have fast gravity waves, but to set the $\mathrm{Fr}$ high enough where the CFL requirement is not restrictive. The CFL requirement is

$\mathrm{CFL}=\left|\delta_{\mathrm{I}}^{2} u+\delta_{\mathrm{I}}^{2} / F r\right|(d t / d x)$. This is also unsuccessful since slow gravity waves interact incorrectly with the other modes. The condition, $\delta_{\mathrm{I}}^{2} / F r>.3$, must be met, which is why $F r=0.001$ is used in all the simulations.

\subsubsection{Effects of Sloping Bottom Bathymetry}

In the past, the study of wind-driven circulation has been dominated by theory based on models with flat bottoms and vertical sidewalls (Jackson et al., 2006). The impact of the coastline orientation and bottom topography on the western boundary current patterns has not yet been sufficiently addressed (Ozgokmen et al., 1997). However, for some time now there has been belief that the response of the ocean may be strongly controlled by the shape of the ocean bottom. Features, such as Gulf Stream separation, meandering, and variable transport may all be related to topographic effects (Holland, 1967).

The success of Stommel's model (Stommel, 1948), which describes the important features of ocean circulation using the simplest model possible, has left the impression that bottom topography effects are not crucial to accurately modeling wind-driven gyres. Stommel's model is a wind-driven, flat-bottomed, barotropic, rectangular basin with linear flow and only linear bottom friction. This model shows that the beta plane is necessary to produce western intensification. To further support the idea that topography is unimportant, Munk's model (Munk, 1950) is solved under the assumption that the interior flow does not penetrate deep enough to even reach the bottom. Even though no reasoning is given for the assumption, the results are assumed valid since the solutions matched expectations. There have been several other studies since that acknowledge the ideas initiated by Stommel and Munk while simultaneously accentuating the need to understand more complex topographic effects (Hughes and deCuevas, 2001).

The goal in this work is to look at how the sloping bottom affects the parameter study of a wind-driven, single gyre in a square basin. Each of 
the cases run to compare against the Fox-Kemper results is also run with the effects of sloping bottom. The equations used to apply sloping bottom effects are

$$
\begin{gathered}
\frac{\partial \eta}{\partial t}+\delta_{I}^{2} \nabla \cdot((\eta+H-b) \mathbf{u})=0 \\
\frac{\partial \mathbf{u}}{\partial t}+\delta_{I}^{2} \mathbf{u} \cdot \nabla \mathbf{u}+\left(\frac{1}{P l}+y\right) \hat{\mathbf{k}} \times \mathbf{u}=-\frac{\delta_{I}^{2}}{F r^{2}} \nabla \eta+\delta_{M}^{3} \nabla^{2} \mathbf{u}-\frac{1}{\pi} \cos (\pi y),
\end{gathered}
$$

where $\eta$ is the variation of the sea surface height from the mean height, $H$, and $b$ is bathymetry. The exact equation used to define the bottom boundary is

$$
b(x, y)=1-\left(0.07+0.93\left[1-\exp \left(-400(x-0)^{2}\right)-\exp \left(-400(x-1)^{2}\right)\right]\right),
$$

which is constant in the $y$ direction. A $y$-slice is plotted in Figure 7 and shows how the bottom is flat in the interior of the domain, but the bottom smoothly slopes up along the east and west boundaries similarly to a ocean basin with continents on either side.

It is evident from Figure 5 that there is a difference in the circulation pattern between a flat ocean bottom and a sloping ocean bottom. This difference can be explained through the understanding of a simplified version of the shallow water equations called the potential vorticity equation. Starting with the dimensional shallow water equations with numerical viscosity and wind forcing and taking a curl gives the following potential vorticity equation,

$$
\frac{D}{D t}\left(\frac{\zeta+f}{h}\right)=\frac{F}{h}
$$

where $\zeta=\mathbf{k} \cdot \mathbf{w}^{\star}, \mathbf{w}^{\star}=\nabla \times \mathbf{v}, \mathbf{v}=(u, v)$ (the horizontal velocity components), and $h=H+\eta$ is the total depth of the ocean. $F$ represents friction and forcing. The term, $(\zeta+f) / h$, is called the potential vorticity in a rotating system and is a conserved quantity. The $\zeta$ is the relative vorticity with $f$ being the vorticity that the fluid has by virtue of the background rotation. However, if the relative vorticity is neglected and potential vorticity is dominated by the rotation of the earth, then potential vorticity is simply $f / h$. In the flat bottom case, the potential vorticity contours are simply horizontal lines since there is no $x$ variation in the potential vorticity. However, for the sloping bottom case, which has variations in the $x$ direction, the potential vorticity contours converge to the southwest corner of the basin and have strong $x$ variations. 
For the flat bottom case, the streamfunction is advected westwards along the $f / h$ contours, spreading diffusively as it goes. The western boundary layer is created as a consequence of the $f / h$ contours colliding with the western wall and needing to satisfy no slip boundary conditions. For the sloping bottom case, the streamfunction is advected pseudowestwards, i.e. along the potential vorticity contours. Just like in the flat bottom case where friction is needed to move the flow in the meridional direction, in the sloping bottom case, friction is necessary for the flow to cross $f / h$ contours. This explains why there is a kink in the southwest corner of the streamfunction solution for a sloping bottom case, which is because the flow is following the potential vorticity contours (Jackson et al., 2006).

Well resolving the kink that is present in the solutions with a sloping bottom is a difficult task when using a uniform grid. However, as seen in the sloping bottom solutions in Figure 8, the adaptive wavelet method automatically adds the necessary resolution to the southwest corner to guarantee an accurate representation of the small scale kink feature. This is a crucial component of modeling this effect.

The importance of the sloping bottom effect is evident from the presence of the kink in the real ocean observations of western boundary currents (Zhang and Vallis, 2007). It is small features like this that motivate the need for adaptive, non-uniform grids in ocean modeling. The results presented here show how accurately and efficiently these results can be obtained using the adaptive wavelet collocation method approach.

\subsubsection{Wavelet Compression}

Figure 8 shows some instantaneous results and grids for two cases when $R e=0.5$, one with a flat bottom and the other with a sloping bottom. The flat bottom case for this particular time uses 4033 points. The effective resolution is 2048 by 2048, which is $4,184,304$ points. Therefore, only $0.1 \%$ of the total points are used, resulting in a $99.9 \%$ compression. In the sloping bottom case, there are 16260 active points, which is only $0.4 \%$ of the points, resulting in a $99.6 \%$ compression. For steady-state results, like a wind-driven gyre, the maximum level of resolution decreases over the course of the simulation. Initially, during the transient time, the highest level of resolution is required. After the solution reaches steady-state, the finest level of resolution is often two or three levels below what it was initially. Therefore, the compression can be calculated based on the highest level of resolution needed over the entire integration time of the problem or can be the highest 
level of resolution of the instantaneous solution. In most of these boundary problems, the compression is so high in either calculation, so the difference is negligible.

\subsection{Test Case III: North Atlantic Simulations}

The third test case is a wind-drive single gyre in the North Atlantic basin. The computations are based on the shallow water model for the non-dimensionalization in Equations 28 and 29. The results are for a low Reynolds number flow, $R e=\delta_{I}^{3} / \delta_{M}^{3}=0.2$. With these parameters, the flow is laminar and steady, but still serves as a useful preliminary test of combining Brinkman and sloping bottom techniques.

The mask is created from data obtained from NOAA's National Geophysical Data Center using GEODAS Grid Translator. The finest resolution available for the domain of interest is used. To create the masking function, $\chi$, the bathymetry data are normalized to ones and zeroes, filtered and smoothed to guarantee numerical stability. Figure 9 shows the masking function for the North Atlantic. For the definition of bathymetry, the same data are non-dimensionalized and smoothed, as seen in Figure 10. As discussed in previous sections, the effect of topography plays an important role in the solution, so the effects of the realistic North Atlantic bathymetry are incorporated.

Figure 11 shows sea surface height results for low Reynolds number ( $R e=$ 0.2). These smooth, laminar North Atlantic simulations demonstrate proofof-concept for running basin-scale boundary currents using realistic continental topography. The low Re is necessary in order for numerical stability and for computational efficiency. The results, however, do show the western intensification of North Atlantic circulation. The solution is missing all the mesoscale eddies and turbulence present in realistic flow.

\subsubsection{Wavelet Compression}

Figure 12 shows the adaptive grid colored by the different levels of resolution for the North Atlantic simulation. The total number of points on the finest level of resolution is $5888 \times 2560$, which is $15,073,280$ points. There are only $0.2 \%$ of the total points active, which comes out to a $99.8 \%$ compression. As can be seen on the grid, the majority of the adaption occurs near the continental boundaries, especially along the western boundary, the only location where the highest level of resolution is used. Once larger $R e$ solutions can be run, the grid will also adapt to mesoscale eddies which will 
be created at various locations where different currents collide. The strength of the adaptive wavelet collocation method will be even more prevalent in these turbulent cases.

\subsection{Test Case IV: 2010 Chile Tsunami}

On February 27th, 2010, a tsunami was generated by an 8.8 magnitude earthquake that occurred near Concepcion, Chile. This tsunami was modeled to round out the fourth and final test case. Since tsunamis are gravity waves, their duration is much more suitable for the shallow water model and for the temporally adaptive grid than the slow Rossby wave and equilibrated gyres.

\subsubsection{Tsunami Generation/Initial Conditions}

Tsunami generation by an earthquake is modeled by sea surface height displacement identical to the vertical deformation of the ocean bottom due to faulting. Usually the horizontal deformation from the earthquake is neglected, unless it is sitting on a steep slope and horizontal displacement is large relative to the vertical displacement. Several assumptions are made when converting the displacement of the ocean bottom to a sea surface height displacement. First, tsunamis are assumed to be long, shallow water gravity waves, which is accurate since the wavelength of a tsunami is much larger than the depth of the ocean. As a result of this assumption, the vertical acceleration of the water particles is neglected compared to the gravitational acceleration. Therefore, the water mass that is lifted is assumed to move uniformly in the horizontal direction. Second, the vertical deformation of the bottom surface of the ocean is assumed to be instantaneous. This is also quite accurate since the phase velocity of a tsunami is much slower than the timescale of the earthquake rupture. Lastly, it is assumed that any horizontal movement in the ocean bottom due to the earthquake is negligible (Tanioka and Satake, 1996).

The most widely used model for tsunami generation is the Okada fault model (Okada, 1985). Earthquake fault data can be obtained from United States Geological Survey (USGS). After retrieving data from a previous earthquake, the fault parameters can be entered into the Okada fault model, which is publicly available on the MATLAB file exchange website. The Okada fault model uses the fault parameters (fault parameters for 2010 Chile earth-

quake are shown in Table 2) to determine what the vertical displacement of the bottom of the ocean is for a particular earthquake using analytic functions derived from theory of a surface deformation induced by an arbitrarily 
oriented rectangular open-mode dislocation Okada (1985). It is then assumed the water is displaced the same amount as the bottom surface of the ocean and that is how sea surface height initial conditions are set. After entering in the right domain and normalizing, Figure 13 shows the sea surface height initial conditions and the data used to generate the 2010 Chile Tsunami. As seen in the plot, the earthquake occurred right off the coast of Chile, which is where the displaced sea surface height is shown.

\begin{tabular}{|r|l|}
\hline Fault Parameters & Value \\
\hline Location of Epicenter & $35.846^{\circ} \mathrm{S}, 72.719^{\circ} \mathrm{W}$ \\
Moment Magnitude Scale, $\mathrm{M}_{\mathrm{w}}$ & 8.8 \\
Fault Length & $400 \mathrm{~km}$ \\
Fault Width & $100 \mathrm{~km}$ \\
Dislocation & $15 \mathrm{~m}$ \\
Strike & $16^{\circ}$ \\
Dip & $14^{\circ}$ \\
Slip & $104^{\circ}$ \\
Depth of Fault & $35 \mathrm{~km}$ \\
\hline
\end{tabular}

Table 2: List of 2010 Chile Earthquake fault parameters. Source:USGS (2010)

Realistic Pacific Ocean bathymetry is used for the tsunami simulation. Since the gravity wave speed is strongly dependent on the depth of the ocean, the effects of realistic bathymetry are important for accurately simulating a tsunami. Figure 14 shows the bathymetry used for the Pacific Ocean.

\subsubsection{Results}

The adaptive wavelet method has proven to be best suited to simulating tsunamis among the test cases presented here. Combined with Brinkman penalization for representing the complex continental topology, it is an efficient model. PRINT VERSION: [Figure 15 shows several snapshots of the tsunami traveling through the Pacific ocean.] ONLINE VERSION: [Video 4.4.2 shows the tsunami traveling through the Pacific ocean.] On the left, sea surface height is shown, while on the right is the adaptive grid, colored by level of resolution. The $R e=2000$ for this simulation and uses the nondimensionalization found in Equations 9 and 10. This Re is still too small to be able to compare these results to observations or to other tsunami models, but the high data compression of the wavelet method should allow for much 
higher Re runs in the future, now that the tsunami case has been found most suitable. Near the end, the tsunami waves start to dissipate (due to numerical viscosity). Therefore, a higher Re number simulation needs to be done in order to compare wave heights.

PLACE Vid2.mov HERE.

There are many steps to be taken to improve the adaptive wavelet tsunami model. First, higher resolution simulations need to be carried out in order to allow for a higher Re and for highly inertial flow. Second, most earthquakes have several aftershocks following the initial mainshock. These aftershocks can be added as sea surface height forcing terms to provide a more accurate model of a tsunami, rather than only forcing the tsunami through the sea surface height initial conditions. Third, there is some reflection off the southern numerical boundary, due to the no slip boundary conditions of the region modeled here. Extending the domain and the masking function to include Antarctica, would improve the reflections off the southern boundary. Better yet, use a gentle Brinkman to damp waves without reflection. Lastly, improved methods for on-shore effects and wetting and drying algorithms would greatly enhance this tsunami model.

\subsubsection{Wavelet Compression}

The total number of points on the finest level of resolution for the tsunami simulation is $5384 \times 3584$, which is a total of $19,267,584$ points. At any given time in the simulation less than $0.1 \%$ of the total number of grid points is used. This is a compression greater than $99.9 \%$, which shows how well suited a tsunami problem is to the adaptive wavelet collocation method. The grid is adapting the continental boundaries, as well as, the tsunami wave propagating through the domain. The grid adaptation of the tsunami wave is most prevalent at the beginning of the simulation before the wave has felt too much of the effect of the numerical viscosity.

\section{Analysis of Numerical Techniques}

\subsection{Computational Cost of Adaptive Wavelet Collocation Method}

For the adaptive wavelet method, the cost per grid point is approximately three to five times greater than with a standard non-adaptive method Kevlahan and Vasilyev (2005). Therefore, as long as there is a minimum of an $80 \%$

compression, the wavelet method compression outweighs the cost. In all of the test cases presented here the compression is much higher than $80 \%$. Out 
of the cases presented here, the least compression was $94.6 \%$ for the rotating waves on a beta plane case and the most was $99.9 \%$ for the tsunami case.

\subsection{Computational Cost of Brinkman Penalization}

There is added cost associated with the implementation of Brinkman penalization. Depending on the $\delta_{\text {pen }}$ used, the computational cost to resolve and Brinkman boundary may be higher than a traditional boundary wall. This is unavoidable but does contribute to improving the accuracy at the boundaries, so in many cases it is worth the cost. The other aspect of Brinkman penalization is the additional domain space. Adding a Brinkman zone means the computational domain needs to be larger, which also increases the computational cost. However, this can easily be minimized by making the zone as small as possible. It is not cost effective to use Brinkman penalization to define straight boundaries. It is a technique to accurately represent complex, variable geometry boundaries, in which case the added computational cost should be expected.

\section{Conclusions and Future Work}

Numerical model development and proof-of-concept testing is completed for the shallow water model. The adaptive wavelet collocation method is validated using Test Cases I and II. These benchmark problems include rotating waves on a beta-plane and a wind-driven single gyre in a square basin. Both test cases demonstrated the strength of the wavelet method in a qualitative way and the wind-driven gyre test case was also quantitatively accurate when compared against a results from Fox-Kemper and Pedlosky (2004).

The shallow water formulation of Brinkman penalization (Reckinger et al., 2012) was used for representation of complex continental boundaries. Test Case III (North Atlantic simulation) and Test Case IV (2010 Chile Tsunami simulation) combine the adaptive wavelet method and Brinkman penalization and provide insight on the best application of these methods. The short time scales of tsunamis make it an excellent candidate for adaptive simulations, while the complex boundaries are represented with ease using the Brinkman method.

High resolution simulations of all the cases developed using the shallow water model need to be carried out as a next step. Also, there are numerous additions that can be made to improve the accuracy and efficiency of these ocean application simulations. For the tsunami model, there are many 
improvements that can be done immediately, including adding aftershocks, improving the boundary conditions on the open boundaries by either incorporating non-reflecting boundary conditions or by extending the domain, and also adding in on-shore effects. The purpose of this work was to show proof of concept simulations of these two novel numerical techniques.

Chui, C., 1997. Wavelets: A Mathematical Tool for Signal Analysis. SIAM Monographs on Mathematical Modeling and Computation, SIAM, Philadelphia.

Daubechies, I., 1992. Ten Lectures on Wavelets. Number 61 in CBMS-NSF Series in Applied Mathematics, SIAM, Philadelphia.

Donoho, D.L., 1992. Interpolating Wavelet Transforms. Technical Report 408. Department of Statistics, Stanford University.

ETOPO2, 2010. Etopo2v2 global gridded 2-minute database. http://www . ngdc.noaa.gov/mgg/global/etopo2.html. National Geophysical Data Center, National Oceanic and Atmospheric Administration, U.S. Dept. of Commerce.

Fox-Kemper, B., 2004. Wind-driven barotropic gyre II: Effects of eddies and low interior viscosity. J. Mar. Res. 62, 195-232.

Fox-Kemper, B., Pedlosky, J., 2004. Wind-driven barotropic gyre I: Circulation control by eddy vorticity fluxes to an enhanced removal region. J. Mar. Res. 62, 169-93.

Holland, W.R., 1967. On the wind-driven circulation in an ocean with bottom topography. Tellus 19, 582-600.

Hughes, C.W., deCuevas, V.A., 2001. Why western boundary currents in realistic oceans are invisid: A link between form stress and bottom pressure torques .

Jackson, L., Hughes, C.W., Williams, R.G., 2006. Topographic control of basin and channel flows: The roles of bottom pressure torques and friction. Am. Meteorol. Soc. 36, 1786-805.

Kevlahan, K.R., Vasilyev, O.V., 2005. An adaptive wavelet collocation method for fluid-structure interaction at high reynolds numbers. SIAM J. Sci. Comput. 26, 1894-1915. 
Kevlahan, N.K.R., Alam, J.M., Vasilyev, O.V., 2007. Scaling of space-time modes with Reynolds number in two-dimensional turbulence. J. Fluid Mech. 570, 217-26.

Liandrat, J., Tchamitchian, P., 1990. Resolution of the 1D Regularized Burgers Equation Using a Spatial Wavelet Approximation. Technical Report. NASA Contractor Report 187480, ICASE Report 90-83, NASA Langley Research Center, Hampton VA 23665-5225.

Liu, Q., Vasilyev, O.V., 2007. A Brinkman penalization method for compressible flow in complex geometries. J. Comput. Phys. 227, 946-66.

Mallat, S., 1998. A Wavelet Tour of Signal Processing. Academic Press.

Munk, W.H., 1950. On the wind-driven ocean circulation .

Nejadmalayeri, A., 2012. Hierarchical Multiscale Adaptive Variable Fidelity Wavelet-based Turbulence Modeling with Lagrangian Spatially Variable Thresholding. Ph.D. thesis. University of Colorado, Boulder.

Okada, Y., 1985. Surface deformation due to shear and tensile faults in a half-space .

Ozgokmen, T.M., Chassignet, E.P., Paiva, A.M., 1997. Impact of wind forcing, bottom topography, and inertia on midlatitude jet separation in a quasigeostrophic model. J. Phys. Oceanogr. 27, 2460-76.

Pedlosky, J., 1987. Geophysical fluid dynamics. Springer.

Reckinger, S.J., Livescu, D., Vasilyev, O.V., 2010. Adaptive wavelet collocation method simulations of Rayleigh-Taylor instability. Physica Scripta T142, 1-6.

Reckinger, S.M., Vasilyev, O.V., Fox-Kemper, B., 2012. Adaptive volume penalization for ocean modeling. Ocean Dynamics , 1-15.

Regele, J.D., Vasilyev, O.V., 2009. An adaptive wavelet-collocation method for shock computations. Int. J. Comp. Fluid. Dyn. 23, 503-18.

Schneider, K., Vasilyev, O.V., 2010. Wavelet methods in computational fluid dynamics. Ann. Rev. Fluid Mech. 42, 473-503. 
Stommel, H., 1948. The westward intensification of wind-driven ocean currents .

Sweldens, W., 1998. The lifting scheme: A construction of second generation wavelets. SIAM J. Math. Anal. 29, 511-46.

Tanioka, Y., Satake, K., 1996. Tsunami generation by horizontal displacement of ocean bottom. Geophys. Res. Lett. 23.

USGS, 2010. http://neic.usgs.gov/neis/eq_depot/2010/eq_100227_ tfan/neic_tfan_wmt.html. United States Geological Survey, Earthquake Hazards Program.

Vasilyev, O.V., 2003. Solving multi-dimensional evolution problems with localized structures using second generation wavelets. Int. J. Comput. Fluid Dyn. 17, 151-68.

Vasilyev, O.V., Bowman, C., 2000. Second generation wavelet collocation method for the solution of partial differential equations. J. Comput. Phys. $165,660-93$.

Vasilyev, O.V., Kevlahan, N.K., 2005. An adaptive multilevel wavelet collocation method for elliptic problems. J. Comp. Phys. 206, 412-31.

Vasilyev, O.V., Kevlahan, N.K.R., 2002. Hybrid wavelet collocation Brinkman penalization method for complex geometry flows. Int. J. Numer. Meth. Fl. 40, 531-38.

Vasilyev, O.V., Yuen, D.A., Paolucci, S., 1997. The solution of PDEs using wavelets. Computers in Phys. 11, 429-35.

Zhang, R., Vallis, G., 2007. The role of bottom vortex stretching on the path of the North Atlantic western boundary current and on the northern recirculation gyre. J. Phys. Oceanogr. 37. 

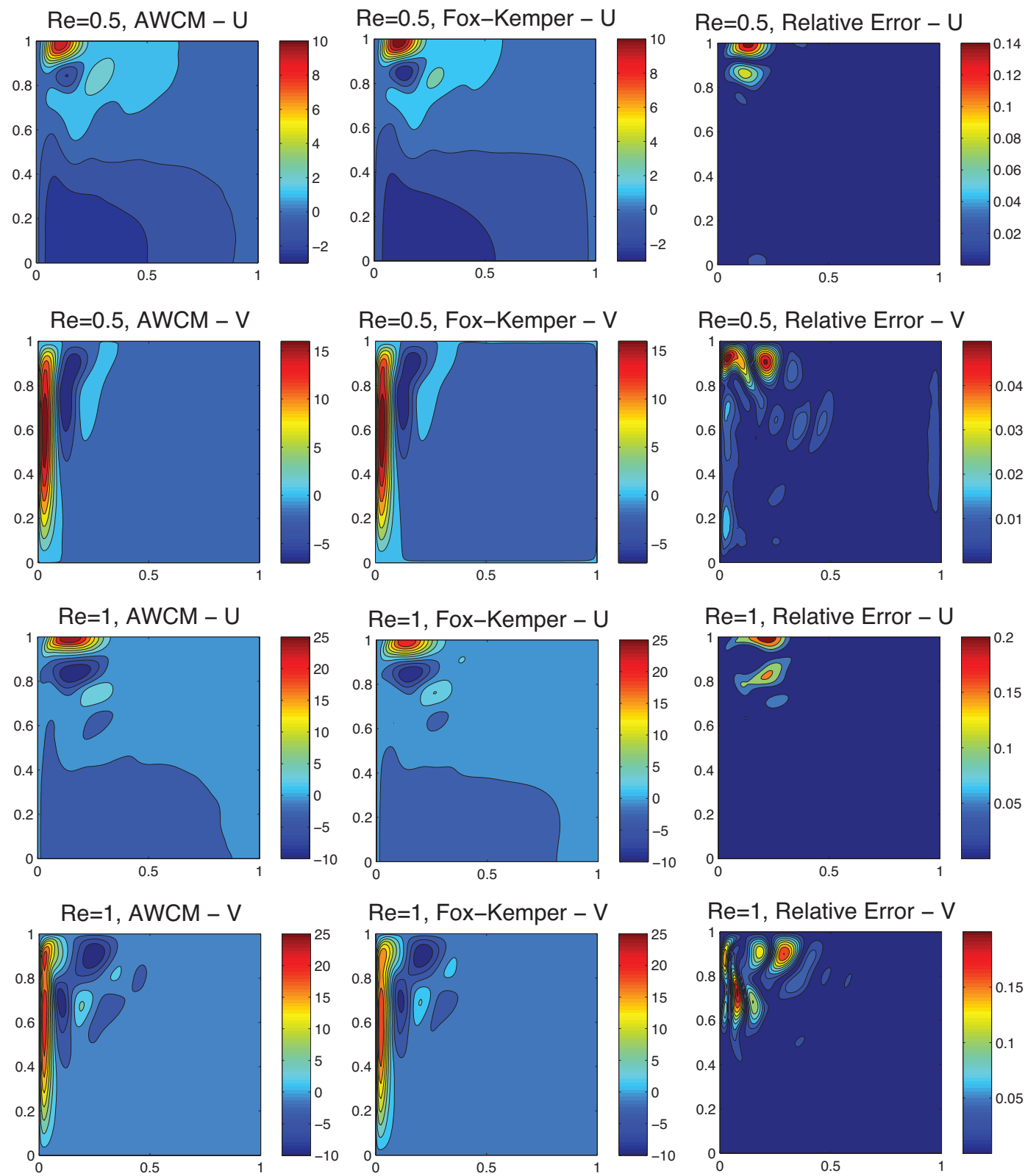

Figure 6: Quantitative Comparison of results from Fox-Kemper and Pedlosky (2004) to cases run using the wavelet method. Plots from left to right show the wavelet method solution, Fox-Kemper solution, and the relative error as function of space. Plots from top to bottom show zonal velocity for $R e=0.5$, meridional velocity for $R e=0.5$, zonal velocity for $R e=1$, and meridional velocity for $R e=1$. 


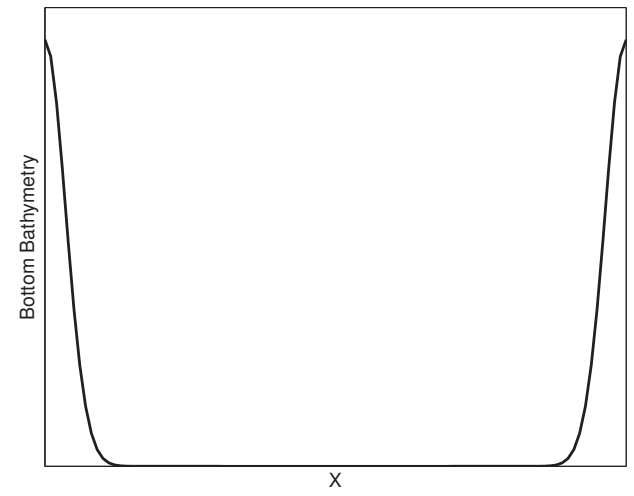

Figure 7: Plot with $x$-axis showing the east-west direction and the $y$-axis showing the variation of the bottom of the ocean. This plot shows the analytical function used to apply a sloping bottom boundary effect. The function is constant in the other horizontal direction (north-south direction).
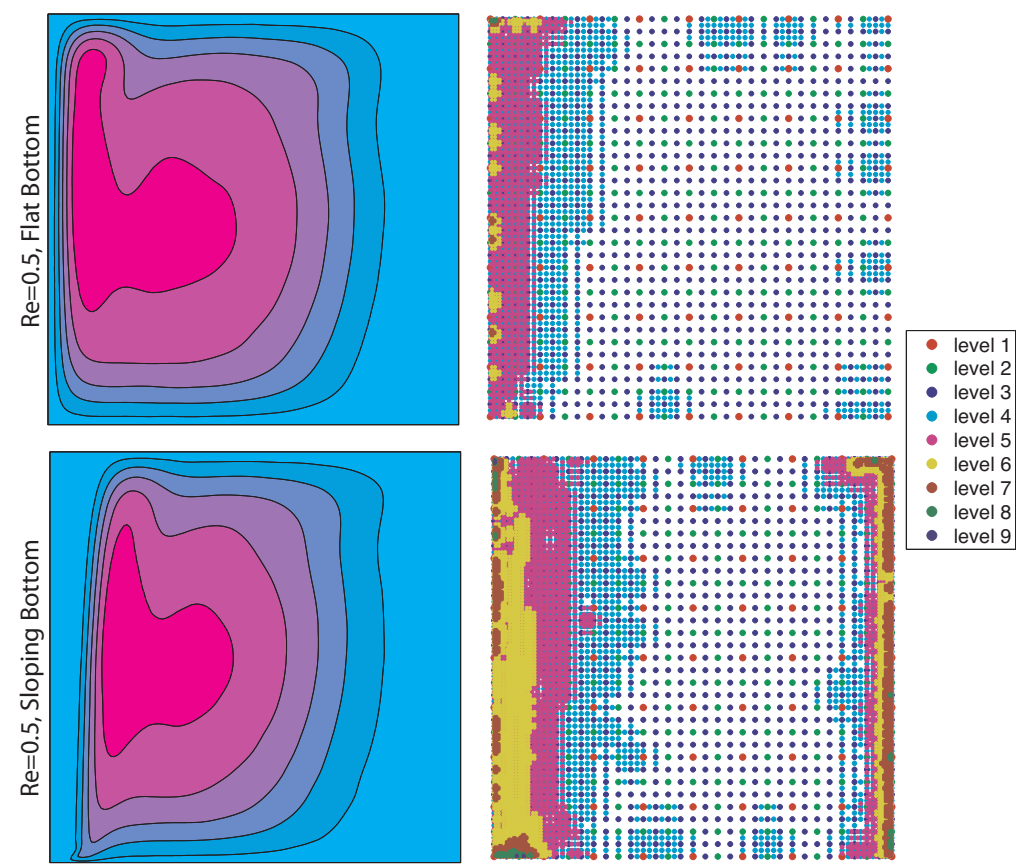

Figure 8: Adaptive grids for two $R e=0.5$ cases. One has a flat bottom and the other has a sloping bottom. These are instantaneous results and grids. 


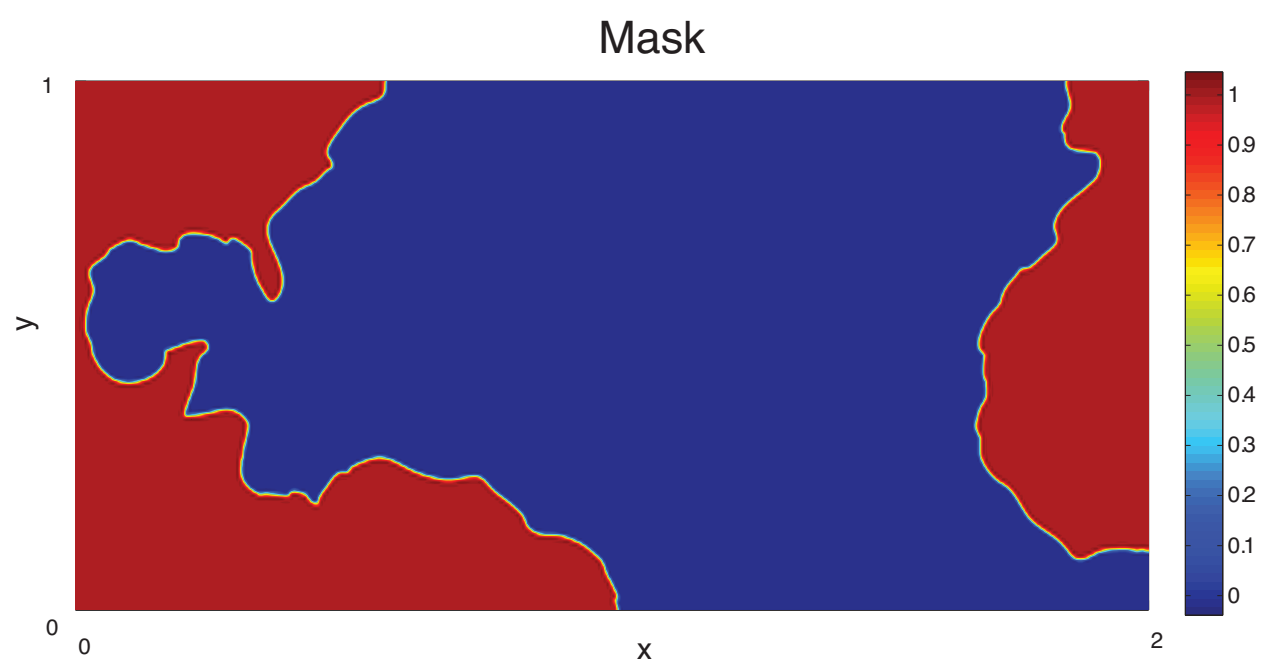

Figure 9: Plot of North Atlantic mask.

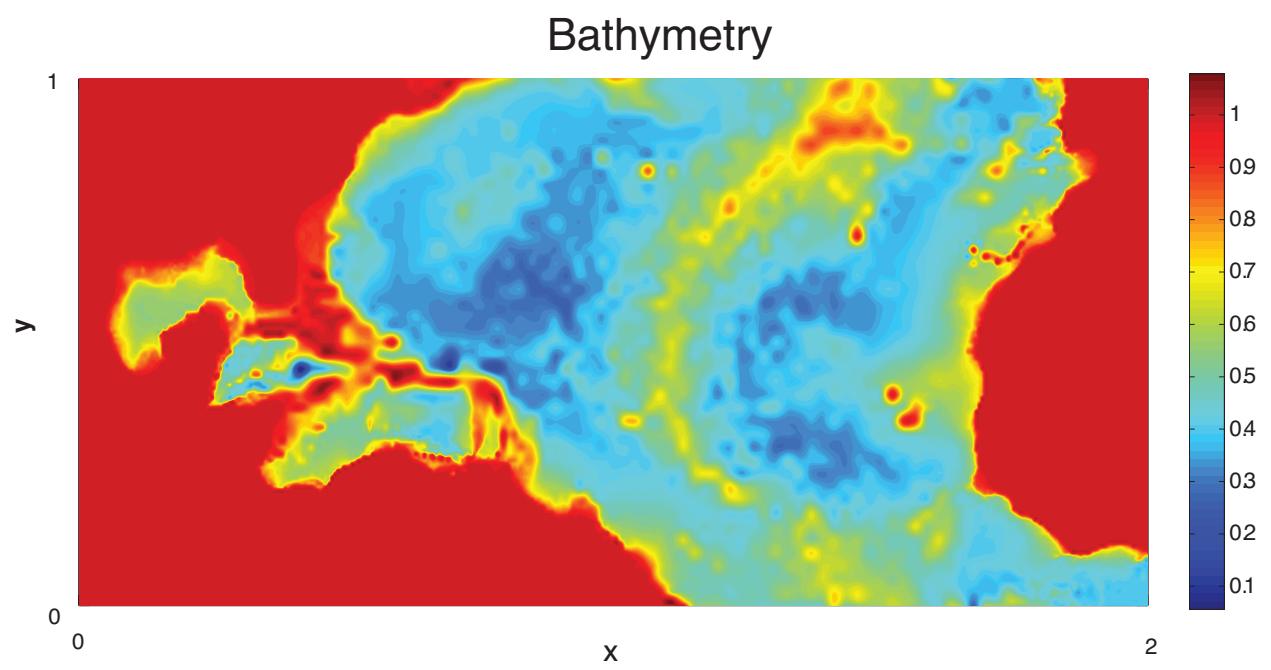

Figure 10: Plot of North Atlantic variable bathymetry data. (ETOPO2, 2010) 


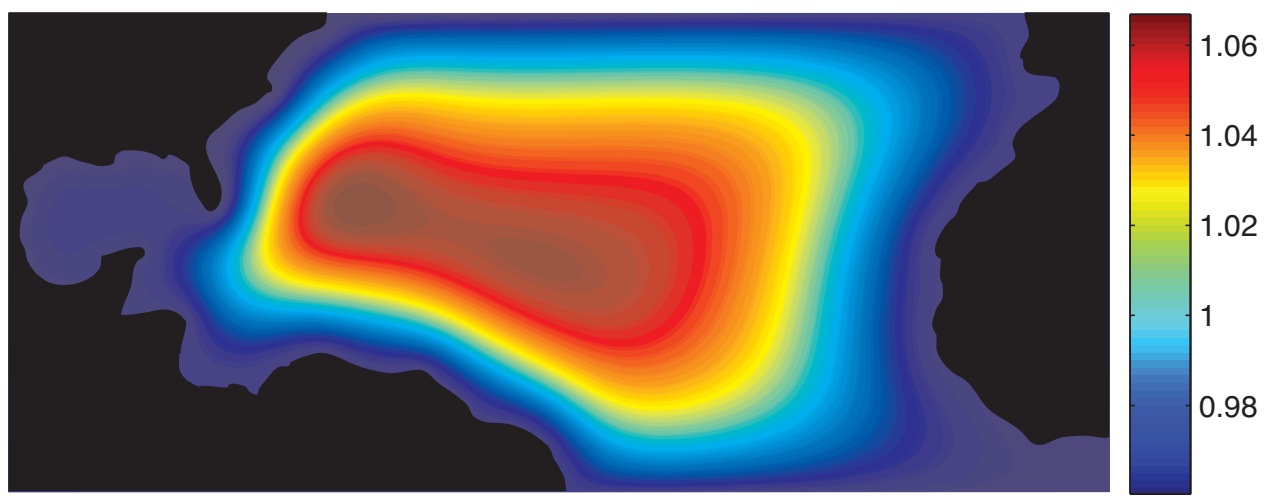

Figure 11: Sea surface height results for a low Reynolds number simulation of the North Atlantic
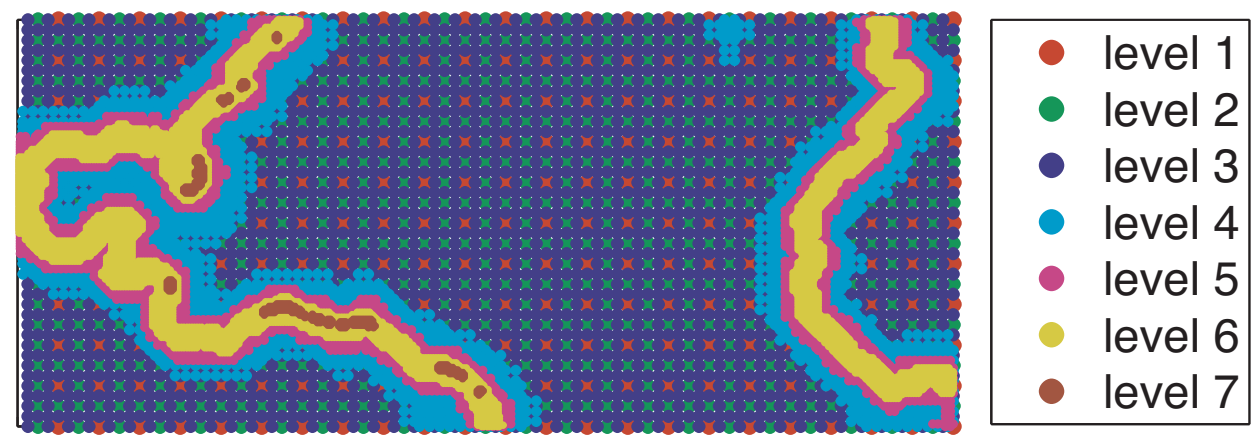

Figure 12: Plot of the adaptive grid for simulations in the North Atlantic. The grid is colored by different levels of resolution. 


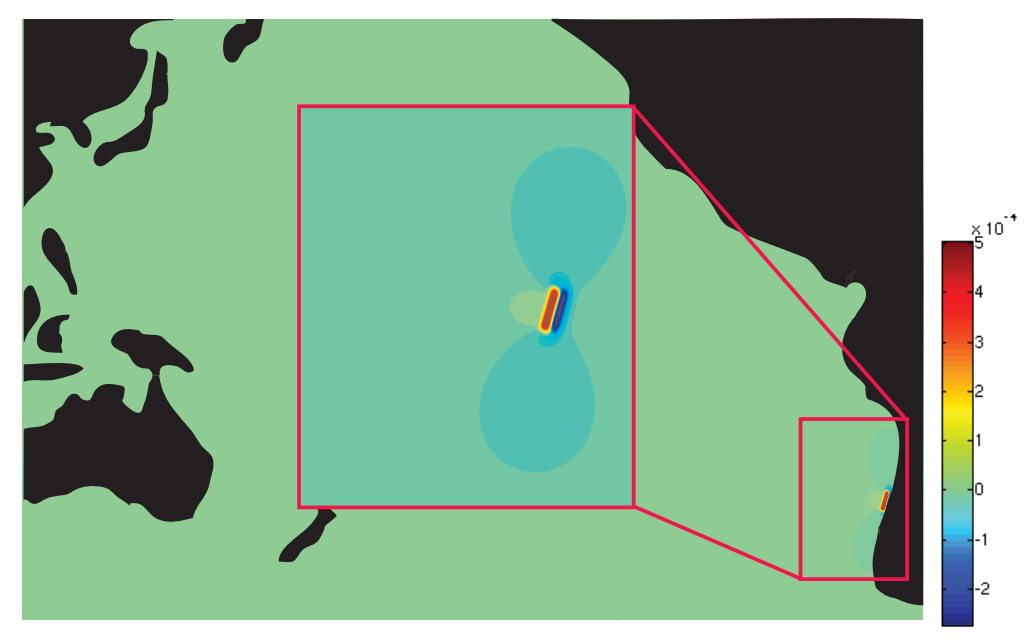

Figure 13: Sea surface height initial conditions for the 2010 Chile tsunami simulation.

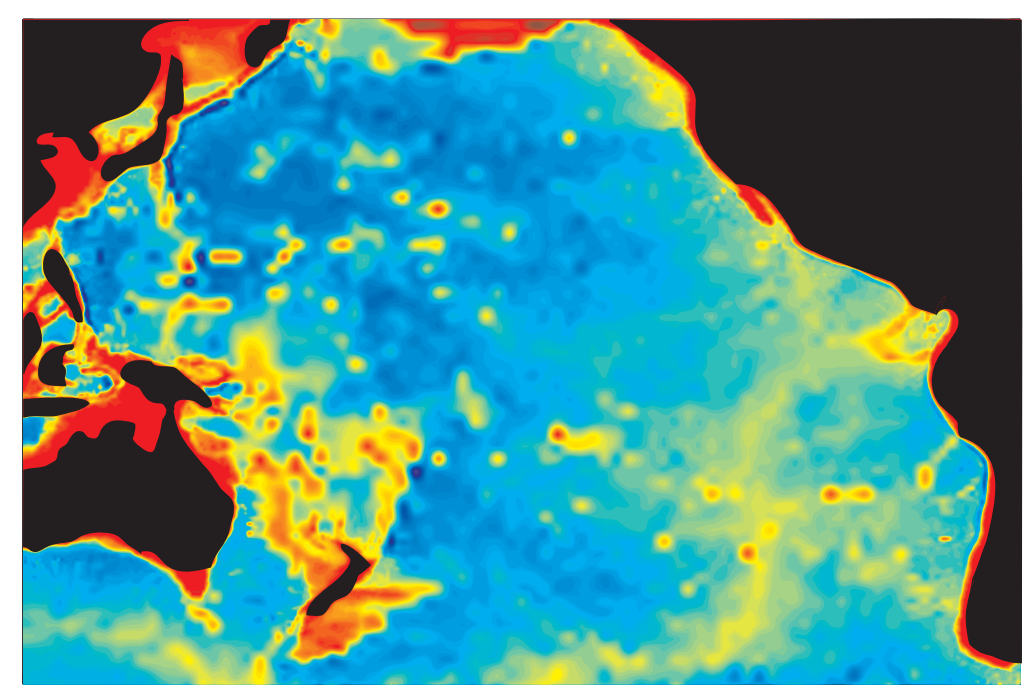

Figure 14: Bathymetry in the Pacific Ocean for the 2010 Chile tsunami simulation. (ETOPO2, 2010) 



- level 5 - level 6 - Bevel 7 level 8 - level 9

Figure 15: Results from 2010 Chile Tsunami simulation, with sea surface height on the left and the adaptive grid (colored by level) on the right. The results are shown chronologically from top to bottom. 